\title{
How much infertility does chlamydia cause?
}

\section{Geoff P Garnett}

Infertility due to obstruction of the fallopian tubes is one of the main severe and lasting consequences of infection with the bacterial sexually transmitted infections gonorrhoea and chlamydia. The disutility and costs associated with infertility and its treatment provide a substantial contribution to the assumed costs of chlamydia in the health economic analyses justifying chlamydia control programmes. ${ }^{1}$ However, infertility is difficult to define and measure. ${ }^{2}$ A lower bound on infertility is provided by medical diagnosis, which requires both that patients seek help and that diagnosis is available. More cases are added if we include all those seeking care for infertility or who believe they are infertile. ${ }^{2}$ Such measures might represent those for whom infertility is a problem, but they will greatly underestimate the true rates of infertility. An alternative is to look at birth histories and periods without any pregnancies, but such methods only readily apply to populations where contraception is not practised. ${ }^{3} \mathrm{~A}$ final method is to estimate the risks of infertility associated with different causes and calculate expected rates. ${ }^{4}$ This approach is generally adopted in health economic analyses ${ }^{5}$ where the proportion of infections leading to disease and the proportion of these that result in infertility is estimated. If these analyses are to be valid we need reliable estimates of the risk of infertility that follows from chlamydia infection.

With developments in chlamydia control, including the use of more sensitive nucleic amplification tests, and screening of those asymptomatic, the effectiveness and benefits associated with the programmes have been challenged. ${ }^{6}$ In justifying intensive programmes it would be useful to have reliable estimates of the risks of salpingitis, infertility and ectopic pregnancy in those who have and have not had chlamydia. To assess the extent to which good estimates exist, Wallace and colleagues (see page 171) carried out a systematic review to see how well the

Correspondence to: Geoff P Garnett, Department of Infectious Disease Epidemiology, Imperial College of

Science Technology and Medicine, St Mary's, Norfolk

Place, London, W2 1PG, UK; g.garnett@imperial.ac.uk excess risk of infertility associated with chlamydia could be estimated. ' They found very little evidence that met their inclusion criteria, which raises two important questions. First, should we believe that chlamydia is an important cause of infertility and, second, how well can we estimate the risk so we can generate improved cost-effectiveness analyses. The key to these questions is the clearly defined exclusion criteria used in the published review. Retrospective case control studies were excluded because these provide odds ratios rather than risk ratios and studies using chlamydia antibody tests were excluded because of their poor sensitivity. However, these methods are the very ones that, along with plausible biological mechanisms, convincingly showed the relationship between chlamydia and infertility. ${ }^{8}$ Additionally, in the absence of prospective cohort studies, such retrospective studies may still provide us with useful results.

The first point to note is that the odds ratio for exposure in diseased versus nondiseased equals the odds ratio for disease in the exposed versus the unexposed, and that this in turn approximates the risk ratio when the prevalence of disease is low. This relationship relies on rates of disease being low and is independent of the prevalence of exposure. To rehearse the justification for these statements consider a study of a single disease and a single exposure. If we denote disease in the exposed as $D e$ and in the unexposed $D u$, and not diseased in the exposed as $\mathrm{Ne}$ and unexposed $N u$, then the exposure odds ratio in those with disease compared to those without disease is $(\mathrm{De} / \mathrm{Du}) /(\mathrm{Ne})$ $\mathrm{Nu})$. This can be rearranged to $(\mathrm{De} / \mathrm{Ne}) /$ $(\mathrm{Du} / \mathrm{Nu})$ : the odds ratio of disease in the exposed versus the unexposed. This is similar to the risk ratio, which additionally includes those with the disease De and $D u$ in the denominators: (De) $(D e+N e)) /(D u /(D u+N u))$. It is clear from this that when the $D e$ and $D u$ are small they contribute little to the denominators and consequently the odds ratio and the risk ratio are similar. The error in calculating the risk ratio from the odds ratio can be estimated if we know the prevalence of disease. Thus, if infertility is low then retrospective case control studies will provide a reasonable estimate of the risk associated with chlamydia. Unfortunately, many of the pivotal studies showing a significant association between infertility and chlamydia antibodies came from populations where infertility was extremely widespread and where errors in the estimate of risk ratios will be great. ${ }^{8}$

In prospective studies, nucleic acid amplification tests provide us with a reliable measure of chlamydia infection. However, in retrospective case control studies, where infertility defines a case, we require a measure of a history of chlamydia infection, not of current infection. Tests for a specific immune response provide such a history. The problem is sensitivity of the tests - a poorly performing test will lead to misclassification bias, and in the case of low sensitivity many of those that should be in the exposed category will be believed unexposed. Such misclassification bias tends to the null model - that is, a relationship will be harder to detect when there really is one. Furthermore, if we know the sensitivity of the test through comparison with a gold standard then we can estimate the actual risk based on that observed using the flawed test.

One concern in the analysis of case control studies is whether confounding variables have been appropriately measured and controlled for. This is especially the case given sexually transmitted infections such as gonorrhoea, syphilis and HIV leading to reduced pregnancy rates. Such a concern also applies for prospective cohort studies. Another area that requires attention is the definition of chlamydia infection associated with a particular risk of infertility. With more sensitive tests, less severe infection associated with fewer complications and sequalea may be detected.

\section{See linked article, page 171}

From the review of Wallace and colleagues, ${ }^{7}$ from experiences in screening programmes ${ }^{9}$ and because programmes are based on cost-effectiveness analyses, ${ }^{15}$ it is clear that more work is required before we can fully justify and appropriately design chlamydia control programmes. However, in this work the case control methods and antibody test that have served us well in the past should not be ignored and perhaps studies where infertility is rare should be a priority. 
Competing interests: None.

Accepted 28 April 2008

Sex Transm Infect 2008;84:157-158.

doi:10.1136/sti.2008.031989

\section{REFERENCES}

1. Hu D, Hook EW III, Goldie SJ. Screening for Chlamydia trachomatis in women 15 to 29 years of age: a costeffectiveness analysis. Ann Intern Med 2004;141:501-13.

2. Boerma JT, Mgalla Z. Introduction. In Women and infertility in sub-Saharan Africa: a multi-disciplinary perspective. Boerma JT, Mgalla Z, eds. KIT, Amsterdam, 2001:13-23.

3. Larsen U, Menken J. Measuring sterility from incomplete birth histories. Demography 1989;26:185-201.

4. Brunham RC, Cheang M, McMaster J, et al. Chlamydia trachomatis, infertility, and population growth in sub-Saharan Africa. Sex Transm Dis 1993;20:168-73.

5. Roberts TE, Robinson S, Barton P, et al. Screening for Chlamydia trachomatis: a systematic review of economic evaluations and modelling. Sex Transm Infect 2006; 82:193-200.

6. Low N. Screening programmes for chlamydial infection: when will we ever learn? BMJ 2007:193-200.
7. Wallace LA, Scoular A, Hart G, et al. What is the excess risk of infertility in women following genital chlamydia infection? A systematic review of the evidence. Sex Transm Infect 2008;84:171-5.

8. Wiesenfeld HC, Cates W Jr. Sexually transmitted disease and infertility. In Sexually Transmitted Diseases (4 ${ }^{\text {th }}$ Edn). In: Holmes KK Sparling FP, Stamm WE, et al, eds. McGraw-Hill, 2008:1511-27.

9. Brunham RC, Pourbohloul B, MAK S, et al. The unexpected impact of a Chlamydia trachomatis infection control program on susceptibility to reinfection. $J$ Infect Dis 2005;192:1836-44.

\title{
Prevention of HIV transmission in the UK: what is the role of male circumcision?
}

\author{
Abigail MacDonald, ${ }^{1}$ Joanna Humphreys, ${ }^{1}$ \\ Harold W Jaffe ${ }^{2}$
}

These two scenarios raise the question of whether male circumcision should be used in the UK as a means to decrease HIV transmission. Lack of circumcision has long been recognised as a risk factor for HIV acquisition in heterosexual men. ${ }^{1}$ This makes biological sense because superficial Langerhans cells, which express HIV-1 receptors, are more prevalent in the male foreskin than in the remainder of the penis. ${ }^{2}$ In addition, decreased keratinisation of the foreskin increases susceptibility to minor trauma during intercourse, potentially aiding the passage of HIV. ${ }^{3}$ Finally, ulcerative sexually transmitted infections, found more commonly in uncircumcised men, are associated with increased rates of HIV transmission. ${ }^{4}$

The biological plausibility of adult male circumcision to reduce HIV transmission has now been shown to have clinical relevance in three recent large randomised controlled trials conducted in sub-Saharan

\footnotetext{
${ }^{1}$ University of Oxford Clinical School, Oxford, UK; ${ }^{2}$ Department of Public Health, University of Oxford, Oxford, UK
}

Correspondence to: Professor H W Jaffe, Department of Public Health, University of Oxford, Old Road Campus, Headington, Oxford OX3 7LF, UK; Harold.Jaffe@dphpc. ox.ac.uk
Africa, in which male circumcision reduced the rate of female-to-male HIV transmission by at least $50 \% .^{5-7}$ Overall, there was little evidence of increased risktaking behaviour in circumcised men. Here we examine whether the results of the African trials are relevant in the UK.

\section{THE ISSUE FOR THE UK}

The yearly number of new HIV diagnoses in the UK has increased by $157 \%$ since 1997. ${ }^{\circ}$ Of the new diagnoses reported in 2006, 12\% were in black African men, most of whom were thought to have been infected heterosexually in Africa, whereas $36 \%$ were in men who have sex with men (MSM). Indeed, 2006 saw the greatest yearly number of newly diagnosed HIV infections in MSM since the start of the epidemic. New prevention strategies for these groups are urgently needed.

\section{SCENARIO 1}

Based on the biological rationale and clinical trials data, the World Health Organization (WHO) has recently recommended that "countries with high prevalence, generalised heterosexual HIV epidemics that currently have low rates of male circumcision consider urgently scaling up access to male circumcision services". Although the hypothetical patient described in scenario 1 resides in the UK, we believe that the WHO guidelines would apply to him and argue that it would be appropriate to offer circumcision to him.

Current National Health Service (NHS) guidelines, however, appear to pertain mainly to paediatric circumcision and generally discourage the procedure. For example, NHS Choices notes that "In the UK, circumcision is only carried out on the NHS in cases where it is medically necessary. It is usually performed as a last resort when other types of treatment have been unsuccessful."10 A similar stance is taken by the British United Provident Association, a major source of private healthcare insurance in the UK (personal communication with Actuarial and Medical Risk Analyst, British United Provident Association, 15 August 2007).

\section{SCENARIO 2}

In their guidelines, WHO made no specific recommendation regarding circumcision for MSM. The US Centers for Disease Control and Prevention also reached no conclusion on this issue for MSM in the United States. ${ }^{11}$ Simple extrapolation from the data on African heterosexual men is clearly inappropriate because MSM engage in a variety of other sexual practices, including anal and oral intercourse, which can be either insertive or receptive. Of these practices, those involving anal sex, particularly receptive anal sex, are most likely to transmit HIV. 
Potentially, the circumcision of MSM who engage in insertive anal intercourse might protect them from infection from receptive partners or, if they are already infected, it might protect their receptive partners from infection. The results of studies on circumcision status and the risk of HIV in MSM are, however, very limited and conflicting. Both a cross-sectional and a prospective study of MSM in the United States found an increased prevalence of HIV in uncircumcised men. ${ }^{12}{ }^{13}$ Neither study was designed to determine the specific sexual practice by which these men became infected. A more recent cross-sectional study of black and Latino MSM in three US cities found no evidence that circumcision was protective in men who engaged in unprotected insertive anal sex. ${ }^{14}$ A prospective Australian trial study also reported no relationship between circumcision status and HIV seroconversion in either the entire MSM study population or those men who denied practising receptive anal intercourse. ${ }^{15}$

Given the lack of data, we would also be unable to make a specific recommendation regarding the patient presented in scenario 2 .

\section{WHAT SHOULD BE DONE?}

First, we believe the NHS and, potentially, private insurers, should examine their existing policies for adult male circumcision. For recent male émigrés from high HIV-prevalence countries in sub-Saharan Africa whose female partners are also from these countries, we believe circumcision should be made available in accordance with WHO recommendations.

Second, to determine definitively whether circumcision could play a role in HIV prevention for MSM, a clinical trial is needed. Major determinants of the feasibility of such a trial would be HIV incidence in the participants and current circumcision rates.

The Health Protection Agency estimated that among MSM attending sentinel genitourinary medicine clinics in London during 2006, HIV incidence was $2.6 \%,{ }^{8}$ a rate generally similar to that seen in the non-circumcised control groups enrolled in the African clinical trials. ${ }^{5-7}$ Whereas these findings might suggest that an MSM trial could be performed with similar numbers of persons as in the African studies, an estimate of HIV incidence for men engaging in unprotected insertive anal sex would be needed for a sample size calculation.

The relatively low circumcision rates of UK men would also facilitate circumcision

\section{Scenarios}

Scenario 1: A 24-year-old HIV-uninfected heterosexual man who has recently emigrated from Kenya presents to a general practitioner surgery in the United Kingdom requesting a referral for circumcision, because several of his friends in Kenya have had the procedure to reduce their risk of acquiring HIV. His partners in the United Kingdom are also recent émigrés from sub-Saharan Africa. What should be the response?

Scenario 2: The presenting patient is an HIV-uninfected 24-year-old sexually active homosexual man from the United Kingdom, also requesting a referral for circumcision to reduce his risk of HIV infection. Should the response be different?

trials. The National Survey of Sexual Attitudes and Lifestyle of 2001 found that only $15.8 \%$ of UK men between the ages of 16 and 44 years had been circumcised. ${ }^{16}$

Of course, such trials would not be ethical without extensive risk-reduction counselling for participants and would not be possible without the willing participation of at-risk MSM. Pilot studies would be needed to determine the likely participation rates. A recent survey of uncircumcised MSM performed in the United States indicated that more than half would be willing to be circumcised if this were shown to reduce the risk of HIV infection. ${ }^{17}$

The primary study endpoint would be the effect of circumcision on HIV incidence for men engaging in unprotected insertive anal sex. We believe this study could be conducted in UK genitourinary medicine clinics. If additional trial sites were needed to recruit a sufficiently large number of participants, they could be identified in countries with a concentrated MSM HIV epidemic and low circumcision rates; eg, in Scandinavia or Latin America.

A second study might also be considered to examine the effect of circumcising previously infected MSM on the risk of infecting persons who are their receptive partners in unprotected anal sex. An analogous study of circumcision for HIV-infected Ugandan men to reduce the risk of infecting their female partners was, however, stopped prematurely.' Preliminary data had shown no evidence of decreased transmission from circumcised men and suggested that men who resumed sexual activity before complete wound healing might pose an increased transmission risk to their partners. Further consultation would be needed to decide if this MSM study should be carried out in the UK.

The UK has the opportunity to lead in revising its male circumcision guidelines in accordance with new African data and to develop data upon which to consider new circumcision strategies for MSM. We believe these opportunities are important and should not be missed.

Acknowledgements: The authors would like to thank A Shepherd, E Turley and K Harvey for their input.

Competing interests: None declared.

Contributors: All authors contributed to the formulation, research and writing of this article.

Accepted 25 January 2008

Published Online First 18 February 2008

Sex Transm Infect 2008;84:158-160.

doi:10.1136/sti.2008.029934

\section{REFERENCES}

1. Moses S, Bailey RC, Ronald AR. Male circumcision: assessment of health benefits and risks. Sex Transm Infect 1998;74:368-73

2. Patterson BK, Landay A, Siegel JN, et al. Susceptibility to human immunodeficiency virus-1 of human foreskin and cervical tissue grown in explant culture. Am J Pathol 2002;161:867-73.

3. McCoombe SG, Short RV. Potential HIV-1 target cels in the human penis. AIDS 2006;20:1491-5.

4. Weiss HA, Thomas SL, Munabi SK, et al. Male circumcision and risk of syphilis, chancroid and genital herpes: a systematic review and meta-analysis. Sex Transm Infect 2006:82:101-9

5. Auvert B, Taljaard D, Lagarde E, et al. Randomized controlled intervention trial of male circumcision for reduction of HIV infection risk: the ANRS 1265 Trial. PLoS Med 2005;2:e298.

6. Bailey RC, Moses S, Parker CB, et al. Male circumcision for HIV prevention in young men is Kisumu, Kenya: a randomised controlled trial. Lancet 2007:369:643-56.

7. Gray RH, Kigozi G, Serwadda D, et al. Male circumcision for HIV prevention in men in Rakai, Uganda: a randomised trial. Lancet 2007;369:657-66.

8. Health Protection Agency. Testing times. HIV and other sexually transmitted infections in the UK: 2007. www.hpa.org.uk/infections/topics_az/hiv_and_sti/ publications/AnnualReport/2007/default.htm (accessed 8 Jan 2008).

9. WHO and UNAIDS. New data on male circumcision and HIV prevention: policy and programme implications. March 2007. www.who.int/hiv/ mediacentre/MCrecommendations_en.pdf (accessed 8 Jan 2008).

10. NHS Choices. Circumcision. When should it be done? http://www.nhs.uk/Conditions/Circumcision/ Pages/When-should-it-be-done.aspx?url = Pages/ What-is-it.aspx (accessed 8 Jan 2008).

11. Sullivan PS, Kilmark PH, Peterman TA, et al. Male circumcision for prevention of HIV transmission: what the new data mean for HIV prevention in the United States. PLoS Med 2007; 4:e223. 
12. Kreiss JK, Hopkins SG. The association between circumcision status and human immunodeficiency virus infection among homosexual men. J Infect Dis 1993:168:1404-8.

13. Buchbinder $\mathbf{S}$, Vittinghoff $E$, Heagerty $P$, et al. Sexual risk, nitrite inhalant use, and lack of circumcision associated with HIV seroconversion in men who have sex with men in the United States. J Aquir Immune Def Syndr 2005;39:82-9.
14. Millett G, Ding H, Lauby J, et al. Circumcision status and HIV infection among black and latino men who have sex with men in 3 US cities. J Acquir Immune Defic Syndr 2007;:46:643-50.

15. Templeton DJ, Jin F, Prestage GP, et al. Circumcision status and risk of HIV seroconversion in the HIM cohort of homosexual men in Sydney. 4th IAS Conference on HIV Pathogenesis, Treatment and Prevention. 22-25 July 2007, Sydney [Abstract WEAC 103].
16. Dave SS, Johnson AM, Fenton KA, et al. Male circumcision in Britain: findings from a national probability sample survey. Sex Transm Infect 2003;79:499-500.

17. Begley $\mathbf{E}$, Jafa K, Voetsch A, et al. Willingness of men who have sex with men in the US to be circumcised as adults to reduce risk of HIV infection. 14th Conference on Retroviruses and Opportunistic Infections; 25-28 February 2007, Los Angeles [Abstract 983].

BMJ Journals editors select an article from each issue to be made free online immediately on publication. Other material is free after 12 months to non-subscribers. Access the Editor's Choice from the home page-or expand your horizons and see what the other BMJ Journals editors have chosen by following the links on any BMJ Journal home page. 